\title{
Abhandlung
}

Benjamin R. Foster* and Andrew R. George*

\section{An Old Babylonian Dialogue between a Father and his Son}

https://doi.org/10.1515/za-2020-0004

Abstract: This article is the editio princeps of a large Old Babylonian clay prism, now in the Yale Babylonian Collection. The text written on it is a long wisdom composition that is hitherto mostly unknown. It begins with a father and son embroiled in an abusive dialogue, and ends with a monologue in which the father looks forward to death and curses his son. The critical edition is accompanied by cuneiform copies and photographs.

\section{The Modern History of the Prism}

The prism published herewith was probably among the first purchases Albert T. Clay made when building up the Yale Babylonian Collection in 1912-13. In a letter to the Secretary of Yale University, dated January 8, 1913, he wrote, "We have the only dialogue of a literary character in Babylonian that is known."1 This must be our prism. Its provenance was probably Larsa, for that was the source of many of Clay's earliest purchases. A photograph of the first home of the Collection (1912-18) shows the prism on the top shelf, left, of the left-hand case, and in the second home of the collection (1918-30), bottom shelf, left, of the left-hand case. ${ }^{2}$ It was not formally accessioned, however, until 1939, when Ferris J. Stephens assigned it the museum number YBC 2394. Unlike the two mathematical prisms accessioned at the same time, YBC 2391 and 2392, Stephens included no description. Albrecht Goetze added, at a later date, "Dialog." Notes found among Goetze's papers show that he worked on the prism, but he evidently abandoned that undertaking in favor of the Old Babylonian omen tablets published in YOS 10 and never returned to it. Placed in storage after the Collection's move to Sterling Library in 1930, the prism

1 Yale University Manuscripts and Archives, Office of the Treasurer, Record Unit 151, YRG 5-A, Series III Box 348 folder 2383.

2 B. R. Foster, Albert T. Clay and his Babylonian Collection, in: B. J. Collins/P. Michalowski (eds.), Beyond Hatti. A tribute to Gary Beckman (Atlanta 2013) 121-35 (pp. 128, 135).

*Corresponding authors: Benjamin R. Foster, Yale University; Email: benjamin.foster@yale.edu

Andrew R. George, SOAS, University of London;

Email:ag5@soas.ac.uk attracted no further interest until the electronic catalogue of the Collection was begun in 1988 with support from the National Endowment for the Humanities. In 2002, Ulla Kasten drew Foster's attention to the prism, and he began work on it. His initial decipherment benefited from several presentations to the Yale Cuneiforum and incorporated readings and corrections that arose in that context, especially suggestions by Eckart Frahm and Enrique Jiménez. In 2018, with support from the Kingsley Fund of Yale University, Andrew George completed decipherment of the prism and prepared the copy published herewith (Figs. 1-7). In the autumn of 2018 the prism was read at the London Cuneiforum and gained from the suggestions of several colleagues. The authors further thank Alberto Urcia and Elizabeth Payne, who collaborated on producing the excellent photographs included here (Figs. 8-11).

\section{The Prism as an Object}

The prism YBC 2394 is $26.5 \mathrm{~cm}$ high and $9.5 \mathrm{~cm}$ wide. Its base is almost square, measuring 9.3 by $10 \mathrm{~cm}$. The four sides originally held eight columns of Old Babylonian cuneiform, continuing onto the base. Side A and the base are well preserved. Sides B and D have suffered extensive losses, especially in cols. iv and vii, and Side $\mathrm{C}$ is missing entirely. The prism is pierced its entire height by a hole approximately $1.7 \mathrm{~cm}$ in diameter. To judge from YBC 2392, an Old Babylonian(?) mathematical prism, in which the hole was pierced after the prism had been made, centered on two diagonal lines drawn from corner to corner on the bottom, YBC 2394 was manufactured first, then pierced for the shaft, and finally removed from the shaft so the writing 
could be continued on the bottom. How a large pointed shaft could be thrust through a moist clay prism without distorting it, how the prism could be kept moist for what was no doubt a long-drawn-out process of writing out the text, and how the shaft could be removed for the final writing on the bottom without damaging the prism remain mysteries of the scribal art. Presumably the purpose of the shaft was to allow the scribe to turn the prism when he got to the bottom of a column in order to begin the next one.

\section{The Content of the Prism}

The text on the prism is an important addition to the genre of wisdom literature that was so productive in ancient Mesopotamia. As already reported by Michael Streck and Nathan Wasserman, ${ }^{3}$ some of the prism's content (here $\S 4$ ) also occurs on an Old Babylonian fragment published by them in 2014 (here MS b), but the composition as a whole is otherwise entirely new.

If we have restored the beginning correctly, a conventional introduction modeled on Sumerian "Instruction" compositions leads the reader to expect a corpus of morally edifying instructions handed down from an old man to his son or junior. In fact, the composition is a parody of such texts. The old man can do no more than give a single piece of advice ( $\S 1$ ), before the younger man interrupts him. What follows is a dialogue in which the young man repeatedly repudiates the beliefs, values and traditions of his elder (§§ 2-27’ff.). At the end of col. iv a long gap intervenes, with the loss of the whole third side of the prism; on the fourth side, col. vii is also badly broken. When intelligible text resumes in col. viii, the old man has launched into a monologue in which he looks forward to outdoing his son in death, and concludes by roundly cursing him.

The dialogue which occupies the first part of the composition is generically related to the Sumerian satirical dialogues set in school contexts, some of which also contain a repudiation of an old man's authority. ${ }^{4}$ The dialogue's abusive tone and the contempt displayed for the son in the closing monologue are reminiscent of "The Scholars of Uruk," an academic Sumero-Akkadian diatribe in which an old scholar also rails against his son. ${ }^{5}$ The generic affiliations of the text are thus well known.

3 M. P. Streck/N. Wasserman, Mankind's bitter fate. The wisdom dialog BM 79111+, JCS 66 (2014) 39-47.

4 E. g. Edubba C, ed. ETCSL t5.1.3, transl. by J. Black [e.a.], The literature of ancient Sumer (Oxford 2004) 277-80.

5 A. R. George, Babylonian literary texts in the Schøyen Collection (Bethesda, Md. 2009) no. 14.

\section{The Interlocutors}

The dialogue's interlocutors are Put-Ishtar and Mannu-utarrissu; in the first part of the composition the former is abbreviated to Putti and the latter often to Mannu-utar. Both names appear to be imaginary. To Put-Ištar "Brow-of-Ishtar" one may offer the distant comparison of ÉREN. IINANNA, the problematic name of the sender of the Sargonic letter ITT 1, 1073, which Konrad Volk suggests might be a nickname for Sharkalisharri. ${ }^{6}$ The implication of this name may, however, be "Frown," "Baleful Glare" (= sa $\hat{g}-k i$ gíd-da)-of-Inanna," as in the incipit of "The Curse of Agade": saĝ-ki gîd-da den-líl-lá-ke $e_{4}$ "Enlil's baleful glare having slain ...". 7

Mannu-utarrissu is perhaps "Who-Made-Him-Prosper?" with the expected answer "his father," or, more likely, a rhetorical question, "Who-Gave-Him-Good-Guidance?" with the expected answer "no one."

As to their identity, in col. viii Put-Ishtar makes the stupefying statement that he is the sage Watar-hasis (Atrahasis), and reveals that he is a contemporary of king Naram-Sin of the Sargonic dynasty and lives in Agade. In $\S 3$ the father implies that his son grew up with and served Naram-Sin's son and successor, Sharkalisharri, who will dissipate what his father built up. Although this accords with the negative judgment on Sharkalisharri in historical omens, ${ }^{9}$ this information is so much at variance with what else is known of Atrahasis in Mesopotamian tradition as to leave the modern reader guessing at the author's intent.

It is worthy of note that the two speakers address each other by name, rather than using kinship terms, and that the son regularly refers to his father using the abbreviation Putti, perhaps disrespectfully.

\section{The Text on the Prism}

The text is inscribed on the prism in a cursive variety of Old Babylonian cuneiform and displays spellings consistent with being written in southern Babylonia. Double consonants and mimation are inconsistently rendered. The text suffers from occasional lipography and errors in sign selection, especially in the last fifteen lines, when the scribe was tired or hasty as he neared the end of his work.

\footnotetext{
6 B. Kienast/K. Volk, Die sumerischen und akkadischen Briefe des III. Jahrtausends. FAOS 19 (Stuttgart 1995) 106.

7 J. Cooper, The curse of Agade (Baltimore 1983) 73.

8 Streck/Wasserman, JCS 66, 45, consider "Mannu-utār-issu 'Who can turn away his arm?"”.

9 B. R. Foster, The age of Agade (New York 2015) 256.
} 
Lines are grouped through the use of rulings, except on the base. These rulings usually, but not always, delimit units of poetry that are self-contained in terms of syntax and sense. The language is relatively plain and unadorned. In the final section, the sequence of curses, the poetry gives way to an elevated prose.

The text is transliterated from the prism, except in $\S 4$ where the following sigla are used:

\section{Transliteration}

Col. i $\S 1$

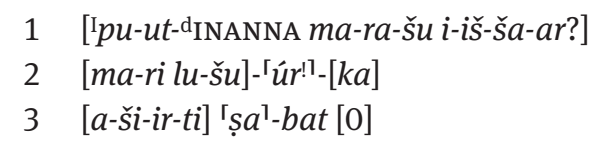

4 [e ta-aq-bi] i-na li-ib-bi a-ši-i[r-tim]

5 [a-na]-ku-mi dam-qá-a-k[u]

6 vacat a YBC 2394

Copy and photographs: Figs. 1-11

b $\quad$ BM 79111+BM 80065

Copy, photographs and edition: M. P. Streck/N. Wasserman, Mankind's bitter fate. The wisdom dialog BM 79111+, JCS 66 (2014) $39-47^{10}$
[ ${ }^{\mathrm{i}}$ Put-Ishtar gave instructions to his son:] “[My son, I will instruct you!] Pay attention to [my instructions!] best!”
[Do not say] in the midst of a lesson, 5 "It is I who am

His son answered him:

Putti,

Being a sage is captivity and oblivion, 10 it is not so precious to Nudimmud as a guardian spirit.

[Though mighty] be the singer's art, wisdom, intelligence, the man who has a protective spirit, what (more need) he do?

11 [lu d]a-nu na-ru-tum ne-me-qum hga- ${ }^{\Gamma} s i^{1-}$ súm

12 [L]Ú ša ši-dam i-šu-ú i-pe-eš mi-nam

13 [e]-li-「ka'-ma a-wa-tum

14 [tap]-ri-id ma-aš-ri-i ù la-ma-sí

$15[a-n] a$ ba-ab ša ši-dam $i$-šu-ú

16 [k]a-mi-is NUN.ME

Is the matter too much for you, [(that) you grew] alarmed at my wealth and good fortune?

The sage kneels ${ }^{15}$ at the door of the one with a protective spirit.

[At] Enlil's command, in the New Year festival?, the ...s pass by.

[(If) a man keeps] sacrificing kids, ${ }^{20}$ his god is [appeased].
17 [i-n]a qí-bi-it den-líl i-Гna a?-ki-tim

$18[\mathrm{x}]^{\top} \mathrm{x} x^{\top} i-b a-u ́$

19 [LÚ it-ta-n]a-aq-qíla-li-a-am

20 [ma-gir] DINGIR-šu
10 W. G. Lambert's handcopy of BM 80065 is now also available, in A. R. George/J. Taniguchi (eds.), Cuneiform texts from the folios of W. G. Lambert (University Park, Pa. 2019) no. 265. 
$\S 3$

$$
21
$$

${ }^{\top} a^{1}-b u$-šu i-pa-al-šu

22 ša a-na șú-uh- ${ }^{\top} r i-k u-n u^{\top}-u ́$

23 at-ta ù šar-r $[a-k] a$

24

25

26

27

ma-ru-šu i-pa-al-šu

a i 44 Ipu-ut-ti ú-ul ta-mu-ur

b i 6 ta-mur Ipu-ut-ti $\rightarrow$

a i 45 i-na na-ri-im mi-lam a-la-kam

b i 6 i-na ÍD mi-lam a-la- ${ }^{\top} a k$-šu

a i 46 6-šu iš-ši-a-am-ma 7-šu it-tur

b i 7 6-šu í[s?]-si-am-ma 7-šu ut-te-e-e[r]

a i 47 sú-un-qum ḩu-ša-huu ni-ib-ri-tum

b i 8 sú-un?-[qu]m hnu-ša-' $a h^{\top}-h{ }^{\prime} u$ ne-eb-ri-tu $\rightarrow$

a i 48 ù $[$ mi]-ti-tum

b i 8 ù mi- ${ }^{-} i^{i}{ }^{1}-[t u]$
His father answered him:

As to what you and your king accomplished in the past for your youth,

As to you who proceeded ${ }^{25}$ in my master's shadow, a fine protection,

Now the stringed instrument is put aside, the song of triumph has died away,

There is no excitement, ${ }^{30}$ celebration is long over, The days are past, the years have come to an end. Brace yourself, get ready for suffering. 35 "The generous hand has gone back into the sleeve." "(If) evil has vanished, a happy man drove (it) away." Your lord will use up the property 40 his father bequeathed him, And what I accumulated you yourself will squander.
His son answered him:

Putti,

Have you not seen 45 the high water coming from the river?

Six times it rose, seven times it receded.

Famine, starvation, malnutrition, and want, 


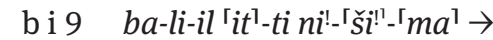

a i 50 la-bi-ri-iš ba-ši

b i 9 la-bi-ri- ${ }^{\top} i \check{S}^{\top} b a-s ̌ i$

a i 51 qá-nu-úi-na a-pi-im

b i $10 q a \hat{a}-n u{ }^{\ulcorner} i-n a^{1} a-p \hat{\imath} \rightarrow$

a i 52 lu-bu-rum i-ma-aq-qú-ut

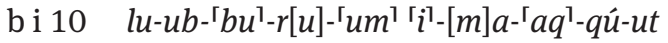

a i 53 ki-ma GI.ÈN.BAR șe-eh-ri

b i 11 ki-ma g[i]-l[u]? 'șe ${ }^{\top}-e h-r u-u[m] \rightarrow$

a i 54 i-il-la-kam a-di $u_{4}$-mi-šu

b i 11 [(i-)il-la-kam] ' $a-d i{ }^{\top} u_{4}-m i-s ̌ u$

a i 55 Ipu-ut-ti ki-i i-na pa-ni-ni

b i 12 Ipu-ut-t[i...]

a i 56 ku-ul-lu-m[a n]i-šu ú-șur-tam

a i 57 am-ra-ti-ma ki-ki-ța ša DINGIR

b i $13 \quad{ }^{\mathrm{G}} \mathrm{GA} / \mathrm{BI}^{\mathrm{T}} \mathrm{x} \times[\ldots]$

a i 58 [p]i-ri-iš-tam te-eš-me

59 qá-bi-mi i-na pi-i $\mathrm{d}$ En-lil

$60 \quad{ }^{\top} a^{1}$-bu ma-ra-am li-ra-am

61 [a]m-mi-ni ma-rum a-ba-am li-ra-am

62 [l]a iq-qá-bi i-na ši-ma-tum

Col. ii

$4 \quad a$-ba-šu li-ih̆-si-'in ${ }^{1}$
$5 \quad a-b u$-šu i-pa-al-šu

6 Ima-「an-nu'-um-ú-tar-is-sú

7 a-na-ku i-na-an-na mi-nam e-ri-iš-ka

8 ip-ra-a-am pi-iš-ša-tam ù lu-bu-ša

9 ki-ša!(tablet: MA)-ma 'iš'-tu șú-uh-rika-ma

10 ța-ar-da-at uk-ku-ša-at

11 ma-ši-a-at i-na li-ib-bi-\{ia $\rangle$
Are mixed in with people and 50 there from of old. The long-lived reed in the canebrake will fall, Just as the young sprig goes on till its time. a

\section{${ }^{55}$ Putti,}

How was it that in times before us, people were shown divine plans?

You are versed in the gods' rites, you heard what was secret.

It was commanded, they say, from Enlil's own mouth: 60 "Father should love son."

Why was "Son should love father" not commanded among things destined to be?

ii And if in good times a father did not protect [his] son, When should a son protect his father in (his) time of suffering?

${ }^{5}$ His father answered him:

Mannu-utarrissu,

I, for my part, what do I ask of you now, Food, oil to groom myself, clothing?

One would think that since you were a child,

10 You were sent away, you were ignored,

You were forgotten, out of [my] heart! 
$\S 6$ ma-ru-šu i-pa-al-šu

Ipu-ut-ti ša it-ti a-wi-lu-tim / mi-tu it-ti DINGIR ba-li-iț da-ri-ša-am

ù ma-ra-am ša i-na É A.BA hu-um-mu-ú $i$-šar LUGAL

$$
a-b u \text {-šu i-pa-al-šu }
$$

Ima-an-nu-ú-tar-is-sú i-de ša DUMU.MEŠ i-na pa-ni-ka

ù ši-ip-ra ša ța-bu ù ni-pi-sà $a-n a-k u[h] a-a s-s a ̀-a-k u$

i-lum iš-[ku-u]n i-na ú-șur-tim ap-lam a-na la NUMUN ha-la-qí-im

ti-de-ma at-ta ma-rum

ma-la ši-ir-ri ù DAM

$a-b a$-šu $\langle l i\rangle-i t$-ṭù-ul

i-na DUMU.MEŠ 5678

ma-an-nu še-er-ra-am ša ța-bu

a-na a-wi-lim li-iš-ru-uk

a-nu-um-ma LÚ ša DUMU la i-「̌̌s ${ }^{\top}-u ́$ na-a-aș it-ti a-hi-šu

$$
36 \quad a-b u \text {-šu i-pa-al-šu }
$$

37 Ima-an-nu-ú-tar

38 Ie-ta-na a-na šar-ri a-hii-šu

39 im-ți-a-am la ma-aṣ-am

40 'DUMU?-ka' ha- ${ }^{\top} a s ̦-b u$ ša' $i$-na SILA $\{\mathrm{x}\}$

41 il-qú-ú i-la-a-am [ú-u]l ir-ši

\begin{tabular}{ll}
42 & ma-ru-šu i-pa-a[l-šu] \\
\cline { 2 - 2 } 43 & Ipu-ut-ti at-ta ti-de \\
44 & Ie-ta-na a-na še-er-ri-im \\
45 & it-t[a-g]i-ša-am ša-di-a-am
\end{tabular}

His son answered him:

Putti,

The one with mankind is mortal, the one who lives forever is with god,

${ }^{15}$ But the king will provide for the son who was a cripple in his father's house.

His father answered him:

Mannu-utarrissu,

I know what sons are to you,

${ }^{20}$ But I, for my part, am mindful of agreeable duty and a job to do.

God granted in the natural order a son lest the family name die out.

You yourself know full well: a son (should have) regard for his father, 25 as much as for children and wife. Among sons, be there five, six, seven, or eight of them, Who (among the gods) might give a man (even one) child who is pleasing?

\footnotetext{
${ }^{30}$ His son answered him:
}

Putti,

What is the course of a 70-gur barge, in distress without a rudder?

See here, a man who has no son 35 is looked down on by his brother.

His father answered him:

Mannu-utar,

Etana was less than, nor did he measure up to, the king his brother.

${ }^{40}$ Your son? is a potsherd: the one who took it from the street, obtained no good luck.

His son answered him:

Putti,

You know full well that Etana

45 Wandered the uplands seeking a child, 
$46 i$-ṣ[ú-ri-iš] $a$-na UKKIN ša DINGIR.MEŠ

47 [ša-me-š $] a-a m i-l i$

48 [a-na a-wi-l]u-tim iš-ku-nu-ni-iš-[šu]

49 š[a-šu $m$ DINGIR.MEš la i-ku-šu-šu-ma

50 še-er-ra-am la iš-ru-ku-šum

51 i-na ši-ma-tim

$\S 11$

52

53

Ima-an-nu-ú-tar

5

ù ša i-na ši-bu-ti-ia

la tap-pa-la!-sà ta-da-bu-ub at-ta

$\S 12$

$m a-r u-\check{u} i-p a-a l-s ̌ u$

59

am-mi-ni ${ }^{\mathrm{I}} p u-u t-t i$

60

61

iš-tu șú-uh-ri-ia-ma it-ti-/-k[a]

62

Col. iii

(gap of 2 or 3 11.)

$\S 13$

[ $a-b u-s ̌ u i-p a-a l-s ̌ u]$

$1^{\prime}$ I[ma-an-nu-ú-tar]

$2^{\prime} a m-m[i-n i \times \mathrm{x} \times \mathrm{x}]$

$3^{\prime} a-n[a \times \times \times \times \times \times]$

$4^{\prime} \quad \grave{u}{ }^{\top} n a-t ̦ u ́ k a^{\top}-\mathrm{x}[\mathrm{x} \mathrm{x} \mathrm{x}]$

5' i-in-ka [ta-na-ši?]

6' ú-ul uk-ta-zi-ib [x (x) x] / x [x x x]

$7^{\prime} \quad$ LUGAL na-e-ri-im ra-bi-[x x x]

8' ma-har be-lí-ia a-wa-ta[m šu-ku-un?]

9' i-na šu-šu-ri-im ' ${ }^{\prime}{ }^{\top}$-[x x x]

$\S 14 \quad 10^{\prime}$

11' Ipu-ut-ti i-na ki-it-[tim]

12' i-ṣa-am uš-mi-du ù x [ x] / i-d[e]

13' iq-li-il-ma ša r[e-su ka-ab-tu?]

14' i-ka-bi-it qá-al-[lum]

Like a bird to the assembly of the gods,

He went up to [heaven].

They ordained for him the lot of mankind,

To him the gods did not approach,

${ }^{50}$ Nor bestow upon him a child,

From among things destined to be.

His father answered him:

Mannu-utar,

You take good care of yourself 55 in ways not available to me,

But that which, in my old age, you have no regard for, you yourself will complain about.

His son answered him:

Why, Putti, ${ }^{60}$ did you yourself say to me, Ever since I was a child with you, I am no son?

...

[His father answered him:]

[Mannu-utar,]

Why ... to ...?

And is it seemly that iii ${ }^{5^{\prime}}$ you covet ...?

Did ... not fawn on ... king of the raging ...?

[Set] the matter before my lord, by setting things straight he [will ...]

10' His son [answered him]:

Putti,

In truth, the insufficient they made numerous and ...

The one [who was important] diminished,

The insignificant one becomes important. 
Ima-an-nu-ú-tar

$18^{\prime}$

$19^{\prime}$

$20^{\prime}$

ma-an-nu ki-ma ka-a-ti

a-ba ba-ni-šu i-țap-pa-lam ma-gal u[štam]-/-ț[i-šu?]

\begin{tabular}{|c|c|}
\hline $26^{\prime}$ & $a-{ }^{-} b u^{1}-s ̌ u i-p a-a l-s ̌ u$ \\
\hline $27^{\prime}$ & ${ }^{\mathrm{I}} \mathrm{r} m a^{\top}-a n-n u-u ́-t a r$ \\
\hline $28^{\prime}$ & $a m-m i-{ }^{\top} n i m{ }^{\top}-m a$ ra-ši $a-k a-[l i] m !$ \\
\hline $29^{\prime}$ & di-ma-tam i-sà-hhu-ur \\
\hline $30^{\prime}$ & $\grave{u}^{\top} \check{s}^{1}{ }^{1}$ DINGIR- ${ }^{\ulcorner} \check{s} u^{\top} i z-z u^{?}-r u-\check{s} u$ \\
\hline $31^{\prime}$ & ú-ra-am 'im?-ți?' \\
\hline
\end{tabular}

i-na zu-um-ri-ka i-na le-[ú]-ti-ka!(tablet: $\mathrm{KU})$

$40^{\prime}$

$$
m a-r u-\check{u} u i-p a-a l-\check{u} u
$$

Ipu-ut-ti ma-an-nu ša a-na me-er-si is-sú-ku li- ${ }^{-} b i^{1}$-tam

ù ša a-na du-um-qá id- ${ }^{\top} d i^{1}-n u$

i-ri-bu lum-nam

Ipu-ut-ti ti-de-e ša a-na-ku

la ap-pa-la-sà-kum qí-bi-it DINGIR-ma

ša dUTU ú-ši-ș[í] pa-ag-ri

41' $\quad a-b u-\check{u}{ }^{\ulcorner} i-p a^{\top}-a l-s ̌ u$

42' [I]ma-an-nu-ú-tar

43' 'ma' at-ta ú-ul LUGAL tap-la-ah

44' ú-ul DINGIR tu-ka-bi-it

45' ú-ul un-ne-nu-ú-a im-qú-tu

46' a-na li-i[b]-bi-ka
${ }^{15}$ ' His father answered him:

Mannu-utar,

The king gave a command concerning you, but god made (you) important.

Who like you, ${ }^{20}$ insulting the father who begot him, [has humiliated him] so much?

His son answered him:

Putti,

Deaf is the one with [a protective spirit], blind is the guardian [angel],

But the one the king has esteemed, ${ }^{25^{\prime}}$ exalts the father.

His father answered him:

Mannu-utar,

Why is it that one who has something to eat seeks about for sorrow?

$30^{\prime}$ But the one whose god has cursed him, is short of a roof (over his head).

His son answered him:

Putti,

Whoever tossed a mud brick into the pudding,

Or returned evil ${ }^{35^{\prime}}$ to one who did good?

Putti,

You know full well that my having no regard for you is the command of a god,

${ }^{40}$ It was Shamash who kept me safe from yourself and your power.

His father answered him:

Mannu-utar,

So! Did you not reverence king, did you not honor god?

${ }^{45}$ ' Have my supplications not fallen upon your heart? 
i-na bi-ri-ni ša ar-ni-[i]m

ub-ba-la-aš-šu a-ra-an-šu li-i[š-š]i

['p]u-ut-ti ma bi-lam [i ni]-iš-ku-un țe $e_{4}-e m-n i$ i-na ma-har ${ }^{\mathrm{d}} \mathrm{UTU}$ qú-ra- ${ }^{\top} d i^{\top}-i m$

$$
a-b u \text {-šu i-pa-al-š[u] }
$$

Ima-an-nu-ú-tar a-di-ni at-ta la tab-ba-aš

ù ša ar-ni-mi iq-b[u-ú] DINGIR ip-țu-ur-[šum?

$$
\text { ma-ru-šu i-pa-al-šu }
$$

a-na-ku Гša ${ }^{\top}$ șú-uh-ri-ia-ma

ú-ul ú-wa-ša-ra-ak-kum

aš-šum-ma iš-tu șú-u[h]-ri-ia-ma

$$
\text { 'ma'-ru-ti la ta-a[h]-še-hnu }
$$

$$
\text { [k]a-mi-il-ka }{ }^{\Gamma} l i{ }^{1 !}-b i
$$

\begin{tabular}{|c|c|c|}
\hline$\S 23^{\prime}$ & $1^{\prime}$ & $\mathrm{x}[\ldots$ \\
\hline & $2^{\prime}$ & ùi-na $\mathrm{x}[\ldots]$ \\
\hline & $3^{\prime}$ & $i d-m i-q[\dot{u} \ldots]$ \\
\hline & $4^{\prime}$ & ša i-qú-ul-l[u... .] \\
\hline & $5^{\prime}$ & $\hat{E}-a-m a \hat{u}-u[l \ldots]$ \\
\hline$\S 24^{\prime}$ & $6^{\prime}$ & $a-b u$-š[u i-pa-al-šu $]$ \\
\hline & $7^{\prime}$ & Ima-an-nu-ú-tar [0] \\
\hline & $8^{\prime}$ & šu-me-lum iṣ-ba-a[t...] \\
\hline & $9^{\prime}$ & ù ša zi-mi-[...] \\
\hline & $10^{\prime}$ & ha-mu-ut-[...] \\
\hline & $11^{\prime}$ & la tu-n[a-...] \\
\hline & $12^{\prime}$ & $i t-t i$ LUGAL $[. .]$. \\
\hline & $13^{\prime}$ & dNin-líl ib-x[...] \\
\hline & $14^{\prime}$ & $i-h a-s ̦ a-a b \times[\ldots]$ \\
\hline
\end{tabular}
a-na a-wi-lu-tim ši-gu DINGIR iš-[ku-u]n

Col. iv

(a long gap intervenes)
His son answered him:

So! Putti, bring it on! Let's set our opinions $50^{\prime}$ before valiant Shamash,

Between us, the one who brings to him wrongdoing, let him bear his punishment.

His father answered him:

Mannu-utar,

55' (Although) so far you have felt no shame, God has ordained penitential prayer for men, And he who said "I am guilty," God has absolved him.

His son answered him:

${ }^{60} \mathrm{I}$, for my part, from my childhood concede to you (nothing),

For the very reason that from my childhood you did not want me as a son,

My heart is angry with you now.

[His son answered him:]

[Putti,] ... and in ... they grew fine ... He who pays heed ... iv ${ }^{\prime}$ did Ea himself not ...?

His father [answered him:] Mannu-utar, The left hand seized ... and the one ${ }^{10^{\prime}}$... suddenly ... Do not ... with the king ... Ninlil ... snaps off ... 
ma-ru-šs $[u$ i-pa-al-šu $]$

$16^{\prime}$

$17^{\prime}$

$18^{\prime}$

$19^{\prime}$

$20^{\prime}$

$21^{\prime}$

$22^{\prime}$

$23^{\prime}$

$24^{\prime}$

$25^{\prime}$

$26^{\prime}$

$27^{\prime}$

$\S 26^{\prime}$

$28^{\prime}$

$29^{\prime}$

$30^{\prime}$

$31^{\prime}$

$32^{\prime}$

$33^{\prime}$

$34^{\prime}$

$\S 27^{\prime}$

$35^{\prime}$

$36^{\prime}$

$37^{\prime}$

$38^{\prime}$

$39^{\prime}$

40' a[m-mi]-ni qáa-x[...]

$41^{\prime} \quad \mathrm{x}[\mathrm{x}(\mathrm{x})] i r^{2}$ [...]

$42^{\prime} \quad t a^{?}-[. .$.

43' te/nim?-ha [...]
${ }^{15^{\prime}}$ His son [answered him:]

Putti,

You heard ... before you ... these fears ...

They complained in ... ${ }^{20^{\prime}}$ will not the king mention ...? And the one whom the king ... let him belittle ...

Putti, you yourself [know:] the one whom a father mentions, ${ }^{25^{\prime}}$ a son belittles.

Now his protective spirit ... passes by ...
His father answered [him:]

Mannu-utar,

$30^{\prime}$ So! Let him [honor] his protective spirit, let him extol his king ...!

As for me who begot [you,] never did my supplications [fall] on [your] heart.

\section{5' His son [answered him:]}

Putti,

By not ... will your mind remember? One would suppose they do not ... before the valiant gods ...

(bottom edge) 
Cols. v and vi destroyed

Col. vii

\begin{tabular}{|c|c|}
\hline $1^{\prime}$ & $\ldots] \mathrm{x}[\mathrm{x} \mathrm{x}]$ \\
\hline $2^{\prime}$ & $\ldots-a] m i h-\mathrm{x}[\mathrm{x}]$ \\
\hline $3^{\prime}$ & ...] x NUN.ME $[(\mathrm{x})]$ \\
\hline $4^{\prime}$ & ...] $i-\operatorname{ta}-\operatorname{ar}[(\mathrm{x})]$ \\
\hline $5^{\prime}$ & ... $] \mathrm{x}$-pi-a-am NUN.ME x [(x)] \\
\hline $6^{\prime}$ & $\ldots q u ́]-{ }^{\top} r a^{7}-d u m[0]$ \\
\hline $7^{\prime}$ & ...-a]m pa-gàr-šu \\
\hline $8^{\prime}$ & $\ldots] \times$ še-mu-um-m[a] \\
\hline $9^{\prime}$ & $\ldots] u \check{\text {. }}$-š $a-a b$ \\
\hline $10^{\prime}$ & [ $\quad$ x x-š $] u i-p a-a l-s ̌ u$ \\
\hline $11^{\prime}$ & [...] x x-ti?-ma bi-tim \\
\hline $12^{\prime}$ & $\ldots] \mathrm{x}$ \\
\hline & (gap) \\
\hline $17^{\prime}$ & ...] ma-ru-t $[\mathrm{im}]$ \\
\hline $18^{\prime}$ & ...] x-ti-ka \\
\hline $19^{\prime}$ & ...] x-a-tim \\
\hline $20^{\prime}$ & ...]-li-il \\
\hline $21^{\prime}$ & ...] x-a-tim \\
\hline $22^{\prime}$ & ...]-ka ma-an-n[u?] \\
\hline
\end{tabular}

$\left.23^{\prime} \quad \ldots ..\right] \mathrm{x}-b i-s ̌ u$

Col. viii

\begin{tabular}{|c|c|}
\hline $\begin{array}{l}1^{\prime} \\
2^{\prime}\end{array}$ & $\begin{array}{l}\ldots . .{ }^{\Gamma} \operatorname{tim}^{1} \\
\ldots . .]-t e-s ̌ u\end{array}$ \\
\hline $3^{\prime}$ & ...] $a-n a-k u$ \\
\hline $4^{\prime}$ & $\ldots ..] \mathrm{x} l a^{?}$ \\
\hline $5^{\prime}$ & {$[l i-i m-d] a-l a$ i-na-a-a } \\
\hline
\end{tabular}

... I myself ... ${ }^{5^{\prime}}$ [let] my eyes fill with [tears.]

Let his [...] take me along. [I will] approach death and turn back to clay.

Now, after the death of Naram-Sin my lord, ${ }^{10}$ I look about for my place of rest, Where neither son nor wife will weep for me,

$7^{\prime} \quad[l u-u k]$-šu-sú mu-ta-am-ma

8' [lu-t]u-úr a-na ți-iț-ṭi

9' [i-na]-an-na EGIR na-ra-am-sin be-lí-ia

10' [ma]-a-a-li a-sà-hu-ur

11' a-šar DUMU ù DAM la i-ba-ku-ú

12' i-na șe-ri-ia 
a-šar ni-šu la i-ma-ra pa-ni-ia

ù i-bi-ir ta-li-mi-ia

la i-qá-bu-ú wu-a-a a-hi tu-uk-la-at-/-ni

a-ma-at-ma i-na li-ib-bu

a-lim a-ga-dèki É ša e-ru-bu-šu

ú-ul ú-šar-ša ba-ba-am

e-li-ma ki-ma e-ta-na

ša-mu AN-nim

ur-ra-ad-ma ap-sí-\{a\}-šu

ú-ul i-lam-ma-ad ma-ma-an

ú-ul qar-ra-da-ku NUMUN me-a-am-li!?

e-ru-um- $\langle m a\rangle$ a-na kur-nu-gi ${ }_{4}$ dereš-ki-gal $a$-ša-ka-an qú-ur-di

$a-s ̌ a-k a-a n-m a$ qú-ur-di i-na KUR i-na pi-i ni-ši di-ši-a-tim

[n]i-šu di-ši-a-tum i-da-bu-ba [da]-li-il qú-ur-di-ia

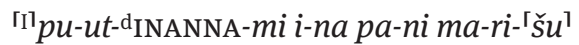
i-te-li ša-me-ša-am

ú-ri-id-ma ap-si-šu-um ú-ul i-ba-aš-ši a-me-er-šu

ú-ša-aq-ba wa-ar-ki-a-tim ni-ši te-ni-ši-tim

${ }^{{ }_{\mathrm{I} l}} p u$-ut-d INANNA-mi NUN.ME wa-tar-hya-si-

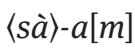

ŠE.NUMUN ú-ma-nim i-na SAHAR KUR bu-lu-ul

ša-ti iš-tu wa-ar-ki-ia-ma ni-šu a-a im-ḩu-ra zi-\{hi\}-im-šu

ša ia-ti i-pu-ša-an-ni ma-rum ki-a-am li-pu-uš-šu a i-pu-uš É i-na la-le-šu

' $a^{1}$ ir-ši ma-ra-am-ma ze-ru-šu

li!(tablet: LA)-ih-li-iq

li-zu-ur-šu-ma a-lum ' $a-g a^{\top}-d e^{\mathrm{ki}}$
Where people will not see my face, Nor will my boon companion say, 15' "Alas for my brother we trusted in!"

I shall die in the city Agade, for the house that I enter I will provide no door.

Shall I go up, like Etana, ${ }^{20}$ into Anu's heaven?

Shall I go down towards the Apsu?

No one will know!

Am I not a valiant warrior, seed of the Hero?

I will enter Ereshkigal's Land of No Return, ${ }^{25}$ I will establish my valor (there),

I will establish my valor in the netherworld, in the speech of the teeming peoples,

The teeming peoples will speak praise of my valor:

30' "Put-Ishtar went up to heaven on account of his son,

"He went down to the Apsu, there is no one who has seen him."

I will make future ${ }^{35^{\prime}}$ folk and people say,

"Put-Ishtar, the sage Watar-hasis, descendant of Umanu, is mixed with the dust of the netherworld!" As for that one, after my death, may people shun him, $40^{\prime}$ The one who treated me so, may a son so treat him. May he build no home in happiness for him, May he have no son but may his lineage die out.

${ }^{45}$ 'May the city Agade curse him, the urban center of the land

May [his] father's curses [pursue him.]

May [the] people revile [his precious life?.]

$\ldots$ 
46' [p]i-ri-ik ma- ${ }^{\top} a^{\top}-[$ tim $]$

47' [š] $]$-ma er-re-et $a-b[i-s ̌ u ~ l i-i t$-he-šu $]$

48' [ni]-šu li-iz-[zu-ra]

49' [wa-a]q-ra-[tam na-pi-iš-ta-šu?]

$50^{\prime} \quad[\mathrm{x}(\mathrm{x}) \mathrm{x}] \mathrm{x}[\ldots]$

2 or 3 ll. missing

Base

1

2 [. . .]-a-šu? i-na É.GAL-š[u ...li-šs] $] a-r i-s ̌ u$

3 [a-al-š]u a-ga-dèki é-ul-maš li-zu-u[r-šu-ma qá-at] ' $m a^{\top}-z a-a z i-p a-r i-k u$ a-a iš-ši

4

[be-el-tu]m mu-dam-mi-qá-at a-wa-tim ${ }^{\ulcorner} k i^{\top}-m a$ w[a-ar-d]i li-qal-li-lam a-waas-sú

5 [ma-ḩar LUGA]L-ri ti-ra ù ma-za-za e-li-šu [li]-iš-ba-al-ki-it

6 [dINNIN].NA qá-ri-ti i-la-tim i-na É-šu $i$-ši-[tam] ù sà-ah-maš-tam li-iš-kuun-šum

$7 \quad[\mathrm{x} \mathrm{x}$-i] $]$ ak-lam a il-li-kam e-li-šu li-ru$u b-m a$

8 [i-na bi]-ti-šu la ța-ab-tum li-im-hnu-ur-šu

9 [dki-li-li be]-el-tum mu-ši-ra-at a-pa-at ni-ši di-〈ši $\rangle$-a-tim

10

[di-li-ip-tam e-r] u-tam ù la șa-la-lam li-išku-un-šu

11 [li-ze-e]r-[̌š ${ }^{1}$ l[i-ik-ru]-ú la-an-šu

12 [li-it-ba-l]am-ma și-la-[̌š ${ }^{1} d u$-ú-sú li-țe ${ }_{4}$-er

$13[\mathrm{~d} m] a-m a$ wa-li-ta-šu ba-ni-a-at \{ras.\} šu-mi-šu

14 [l]i-is-ki-ip-šu-ma a-na ŠÀ LUGAL-šu i-na le-mu-tim / li-〈ir)?-de-šu

15 [l]i-țe ${ }_{4}$-er-ma du-ú-sú ba-aš-ta-šu li-hnal$\langle l i\rangle-i q$

16 [i-na] bi-ri-it ah-hii-šu lu-[ú] du-na-mu!(tablet: NU)-um šum-šu

17 [d] a-ru-ru be-el-tum ba-ni-a-at a-wi-lu-tim š[i-ma-t]i-šu a-na le-me-nim li-išku-un

18 [l]i-pu-uš bi-tam a i[š-k]u-nam ú-ru-ba-tim

19 [li-h] $] u$ - ${ }^{\top} u z$ aš-ša ${ }^{\top}$-tam [a ú-ra]-ab-bi ši-irra-am

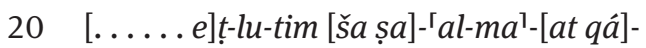
$q \hat{a}$-di-im $\langle l u-u ́\rangle$ e-ri-bu ur-ši-im

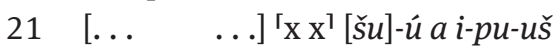

22 [aš-šum... ...la] id-mi-qú-ma $i \check{s}$-bu-su a-ba-šu

$23\left[i-n a\right.$ qi-bi-it $\left.{ }^{\mathrm{d} U T}\right] \mathrm{U}$ da-a-a-nim ša ša-me-e ù er-șe-tim $\cdots$

[May the king,] his [..., have them] escort him from his palace.

May [his city] Agade (and) Eulmash curse [him, that] he not get past the courtiers who block his way (back).

May [the queen] who makes words favorable, make his words contemptible as a [slave's],

${ }^{5}$ May she make eunuch and courtier turn against him before the king.

May [Ishtar], warrior among goddesses, ordain for him disorder and rebellion in his household,

May [...] not pass over him but come in upon him, May he experience nothing good [in] his [ho]me.

May [Kilili], the lady who leans through the windows of the teeming people, ${ }^{10}$ ordain for him [anxiety], insomnia, and sleeplessness,

[May she scorn] him, may his frame shrink, may she [carry] off his protection and take away his virility,

May Mama who bore him, creator of his name, renounce him and denounce him to his king,

${ }^{15}$ May she take away his virility and deprive him of his vitality,

Among his brothers may he be a byword for a fool.

May Aruru, mistress who created mankind, ordain his destiny for evil.

May he build a house (but) hold no housewarming ceremony,

May he take a wife (but) raise no child.

20 [May ... all the] young men [of the black]-headed [race be] visitors to (his) bedroom.

May he himself make no [... ]

[Because... he was not] well-disposed but angry (with) his father,

[By command of] Shamash, judge of heaven and earth, May [his case] never be resolved.

25 Finis 


\section{Exegesis}

$\S 1$. The father alerts his son to the need for modesty when among peers, in accordance with conventional morality.

$\S 2$. The son says that being a sage means that you are a captive to the past and are doomed to be forgotten in the future, so the god of wisdom esteems it less than having a good protective spirit. It is all very well to be accomplished in the arts or intelligent, but what can the man with a good guardian spirit do? The sage kneels at his door, hoping for preferment, whereas Enlil has decreed that the man with a good guardian spirit can pray and make an offering once a year, and his personal god will be content with him.

$\S 3$. The father says that his son enjoyed good times under the protection of the king, but now all that is over. He should prepare himself for bad times to come. The father seems to quote two sayings, one to the effect that the hand of plenty has been withdrawn, another to the effect that it takes a happy person to banish evil, implying that the son is not such a person. Just as his master is wasting his inheritance, so too will the son, and he will be left with nothing.

$\S 4$. The son responds that good times come and go, that want is inherent to the human condition. People grow old and die, young people live out their lives then die too. If the father is such a great sage, knowing hidden things, can he explain why it is ordained that fathers should love sons, but it is not ordained that sons should love fathers? If fathers don't look after their sons in good times, why should sons look after their fathers in bad?

$\S 5$. The father says that to listen to his son, one would think that he had not been a good father. He asks nothing of his son, not even the basics of life.

$\S 6$. The son says that people cannot live forever. The person hindered at home can expect the king's patronage.

$\S 7$. The father says that even if his son is indifferent to what his status implies, he as a father knows what his duty is. Sons are necessary for a family to continue and a son should respect his father as much as he does his own immediate family. One can have many sons but count himself lucky if even one of them is dutiful.

$\S 8$. The son, perhaps mimicking his father $(\S 3)$, quotes for his part two sayings, one to the effect that if even an important person (like his father) is destined to come to grief, that is his fate, and another to the effect that people have children because that is what is expected of them, not because they care about them.
$\S 9$. The father answers that even Etana, the first king, was inferior to his brother, who was presumably not a king, because he had no son of his own and could not found a dynasty. Having a son is a risk (§ 7) but adopting one is even more of a risk.

$\S 10$. The son answers that even if Etana did everything he could to have a son, even going up to heaven and witnessing the gods in assembly, the gods willed that he remain a mortal and have no son, so that was his destiny.

$\S 11$. The father says that the son is better off than he was, and when his time comes to be old, he will complain too, even if he doesn't care about the problems of his father's old age.

$\S 12$. The son asks why his father repudiated him, even as a child.

$\S 13$. Too broken to construe with confidence.

$\S 14$. After a gap in the text, the son is saying that in the way of things one who was important, meaning his father, loses importance, whereas one who was not, meaning himself, becomes important.

$\S 15$. The father says that kings propose, but gods dispose. His son's attitude towards him is not the natural way of things, but unheard of.

$\S 16$. The son says that even when the protective spirit of a person disadvantaged in his family background (as in $\S 6: 15-16$ ) is blind to his needs, if he finds royal favor, (if the text is correctly restored) he brings prestige to his father too.

$\S 17$. The father wonders, rhetorically, why a person who is well off still looks for something to complain about. If god turns against him, then he will really suffer.

$\S 18$. The son responds that no one returns evil for good, implying that if he is not grateful to his father he has no reason to be: he is returning evil for evil. In fact, his disregard for his father was right, and the god of justice himself saved him from his father's personality and authority.

$\S 19$. The father asks if his son in fact has no respect for king or god, does nothing move him?

$\S 20$. The son responds by challenging his father to join him in presenting their cases before Shamash, god of justice.

$\S 21$. The father responds by saying that it is never too late to confess wrongdoing and receive absolution from one's god. 
$\S 22$. The son responds that he is justified in his anger at his father, since his father never wanted him as a son.

$\S \S 23^{\prime}-25^{\prime}$. Too broken to construe with confidence.

$\S 26^{\prime}$. The father seems to be resigned to his son's disobedience.

$\S 27^{\prime}$. Too broken to construe with confidence.

Col. viii and Base. After a long gap, the father says he wants to die and disappear, now that king Naram-Sin is dead. No one will know what happened to him and there will be no place people can go and mourn for him. He reveals himself to be the sage Atrahasis, descendant of Umanu (Oannes). People will speak well of him after his death, but despise his son. He then curses his son, concluding the text.

\section{Textual notes}

i 1: The restoration follows the pattern "PN instructs his son," which occurs in the introductions to two Sumerian compilations of instructions, the Instructions of Shuruppak: šuruppakki-e dumu-ni-ra na na-mu-un$\mathrm{de}_{5}-\mathrm{de}_{5}$ (B. Alster, Wisdom of ancient Sumer [Bethesda, Md. 2005] 57: 6), and the Farmer's Instructions: $u_{4}-u l-$ $\mathrm{uru}_{4}{ }^{\text {ru }} \mathrm{dumu-ni}$ na mu-un-de - $_{5}$ a-àm (M. Civil, The Farmer's Instructions [Barcelona 1994] 28: 1).

i 2-3: Restored after the Akkadian translation of the corresponding line of the Instructions of Shuruppak (Alster, Wisdom of ancient Sumer, 57: 8).

i 4-5: Compare Enkidu's similarly boastful declaration (also discouraged) in Gilgamesh I 221: [lu-uṣ?]-ri-ih ina libbi Uruka-na-ku-mi dan-nu (A. R. George, The Babylonian Gilgamesh Epic [Oxford 2003] 550, reading [lultar?]-ri-ih).

i 8: šallūtum is here used figuratively to mean that a sage is confined far from the human race and so, being out of sight, is out of mind.

i 13: elīkāma suggested by Jon Taylor; [x]-li- ${ }^{-} \tilde{s}^{\top}-m a$ is not ruled out.

i 14: The prism uses the sign value tap on three other occasions (ii 57, iii 43', iv 18'). Among other suitable restorations, we also considered [te]-re-et "[(that I have) a] post (which brings) wealth and good fortune?"

i 25: šabihum is here elucidated by the phrase in apposition, tarānim damqim, lit. "a fine canopy," and we therefore connect it with the verb šabāhum "to form a coat" (e. g. of dust); in astrology šabihhu is a technical term for a phenomenon that coats the moon and planets and obscures them.

i 32: This line yields an OB instance of quttu "finished, at an end.” i 33: utahhiz (Dt imperative?) seems to mean "take hold of yourself," a hitherto unattested usage.

i 35-38: $\grave{u}-\grave{u}$ may indicate that these sections are two proverbial sayings.

i 36: For sūnu, see J.-M. Durand, ARM 30 (Paris 2009) 94f. In the Mari documents, sūnu generally seems to be some sort of attachment to a garment, sometimes colored so likely intended to be seen. According to Ana ittǐsu VII iii 2 (MSL 1,99) it is a place on the person where money can be tied; the Old Assyrian sources cited by Durand suggest an intimate place on the body or in the clothing where small valuables can be hidden, hence "groin" (literally or figuratively). Therefore we understand this to mean that the hand that bestows money has been put back into an inner pocket, with directional suffix -šu.

i 46: Another example of intransitive našûm "to rise" (of tide, river).

i 53: The nominative of MS $b$ is preferred to the oblique case of the prism (influenced by the adjacence of the conjunction kima).

i 62: ši-ma-tum is an error for šimātim; cf. ii 51.

ii 2, 4: The spelling of forms of hașānum with si instead of și or șí is unexpected.

ii 8: ip-ra-a-am is an example of a plene spelling particularly characteristic of scribal practice at Old Babylonian Larsa; see J. Aro, Abnormal plene writings in Akkadian texts, StOr. 19/11 (Helsinki 1953). Another example in this text is $i$-la-a-am (ii 41).

ii 13-14: It is not clear if this couplet means that humans are mortal and gods immortal, like OB Gilgamesh VA+BM iii 4-5: inūma ilū ibnû awīlūtam / mūtam iškunū ana awīlūtim / balātam ina qātišunu ișșabtū (George, Babylonian Gilgamesh Epic, 278), or if it alludes to Utnapishtim as the one human being granted eternal life.

ii 20: ni-pi-ZA is an unexpected spelling of nēpeša $(m)$.

ii 35: For the reading of the verb we are indebted to Antoine Cavigneaux.

ii 38-51: This exchange is a unique comment on the well-known story of Etana. The point of the father's remark is that what set Etana apart and made him memorable was that he had no son. The plain sense of ii 38 is that Etana had a brother, though nothing in the extant traditions about Etana mentions one. The mention of a childless man's brother in ii 34-35 favors the former understanding. Mannu-utarrissu's response argues that, try as he might, Etana never had a son, and that was by divine decree; so too his disregard for his own father is by divine decree (iii $\left.37^{\prime}-40^{\prime}\right)$. This suggests that in this context Etana's successor, Balih, as known from the Sumerian King List, was not thought to be his biological son. While it is tempting to restore biltu in the gap at the beginning of ii 48, which 
in the Middle Assyrian Etana story evidently refers to Etana's childlessness (J. V. Kinnier-Wilson, The legend of Etana: A new edition [Warminster 1985] 58), there seems insufficient space. Since the possibility of immortality, as opposed to the usual lot of humankind, is not found in the preserved texts about Etana, one may speculate that the significance of ii 48 is that insofar as Etana went up to heaven, like Adapa, he might have become an immortal if he had partaken of the gods' food and drink, but did not do so. Thereby ii 49 would mean that he was not singled out by the gods for individual immortality, nor was he even granted a child, the usual way, besides fame, that mortals live beyond their own deaths (ii 50-51). There is nothing unnatural, therefore, in Put-Ishtar's disappointment, contrary to what he thinks - that is what has been ordained for him, just as it was for Etana, and he should not complain. The son's final rhetorical question would thereby imply, "Who can fathom the ways of the gods?"

ii 39: The translation assumes la ma-aș-am for $l \bar{a}$ mași'am. Alternatively read imțiam lamassam (la-masà!-am), “was inferior as regards a guardian angel,” i.e., had less good fortune than his brother.

ii 40: Or 'DUMU.SAG", "A firstborn son is a potsherd: the one who took it from the street, obtained no good luck."

ii 46: Not BANŠUR but UKKIN (S. Mirelman).

iii 13': Alternatively ša-r[i-ú-um] "the rich man."

iii 25': Among other restorations, $u$ [l ḩaših] "has no need of a father" was considered, but there is only room for one sign missing at the line's end.

iii 35': ša a-na is understood to be a transposition of ana ̌̌a.

iii 40': ša Šamaš, lit. "he of Shamash,” i.e. the king?

iii 56': This line suggests that performing the šigûprayer was an act of penitence (cf. n a m - t a g "sin” = šegû V R 16 ii 2).

iii 57'-58': This may be a gnomic or proverbial preterit.

viii 23': As a scholar, Put-Ishtar is of Ea's lineage. The divine epithet mamlum is not otherwise given to the patron deity of scholarship, but another decipherment eludes us. The word is written with first syllable broken also in A VIII/1 90: me-a-am-lum (MSL 14, 491). Compare me- $a$ mu-um for mammum in Proto-Diri 497 (MSL 15, 48), and the emended spelling me- $\langle a\rangle-m u$ re-ši-ni "the crowns on our heads" in an early Old Babylonian love poem (PRAK 1, B 472, ed. N. Wasserman, Akkadian love literature of the third and second millennium BCE [Wiesbaden 2016] 150 i $9^{\prime}$ ). Other examples of dissimilation of /a/ into /ea/ are collected by W. G. Lambert, Babylonian creation myths. CM 16 (Winona Lake, Ind. 2013) 469. viii 36': Alternatively, watar hasīsam is an epithet, “surpassing-wise sage," as it is in Adapa A i 8'.

viii 37': On the sage Umanu see M. P. Streck, Oannes, RlA 10 (Berlin 2003) $1 \mathrm{f}$.

viii 39': To avoid the emendation, one could posit a new noun sihmum, cognate with suhhumum "to bully," and read: ay imhurā sihimšu "May people not countenance his arrogance!"

Base 4: Compare the curses in $\mathrm{CH}$ xlix 81-97, where Ninlil is bēltum(NIN) mu-dam-mi-qá-at i-gi-ir-re-ya "the queen who makes rumour of me favourable," and so has the opposite capacity to turn king Enlil against the transgressor.

Base 6: išittam sahmaštam are also a pair in the curses of $\mathrm{CH}$ li 5-6, where they are inflicted on the transgressor by Ishtar of war.

Base 9: On the owl goddess Kilili mušerrat apātim, see P. Lapinkivi, The Sumerian sacred marriage (Helsinki 2004) 233-36.

Base 18: The context suggests that $u r u(b) b \bar{t} t u m$ was a celebration held when a person moved into a new house, formed from the verbal root "to enter," like its Moroccan Arabic equivalent, dakhla, a housewarming party.

Base 22: šabāsum is usually constructed with eli, but here an accusative of respect is deployed. Alternatively,

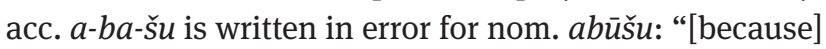
his father was angry."

Base 23-24: Compare, in the causative, a plea to Shamash in an incantation against ghosts (KAR 234 obv. 20): di-ni purussâ(EŠ.BAR) šur-ši "Bring resolution to my case!” 


\section{Drawings}

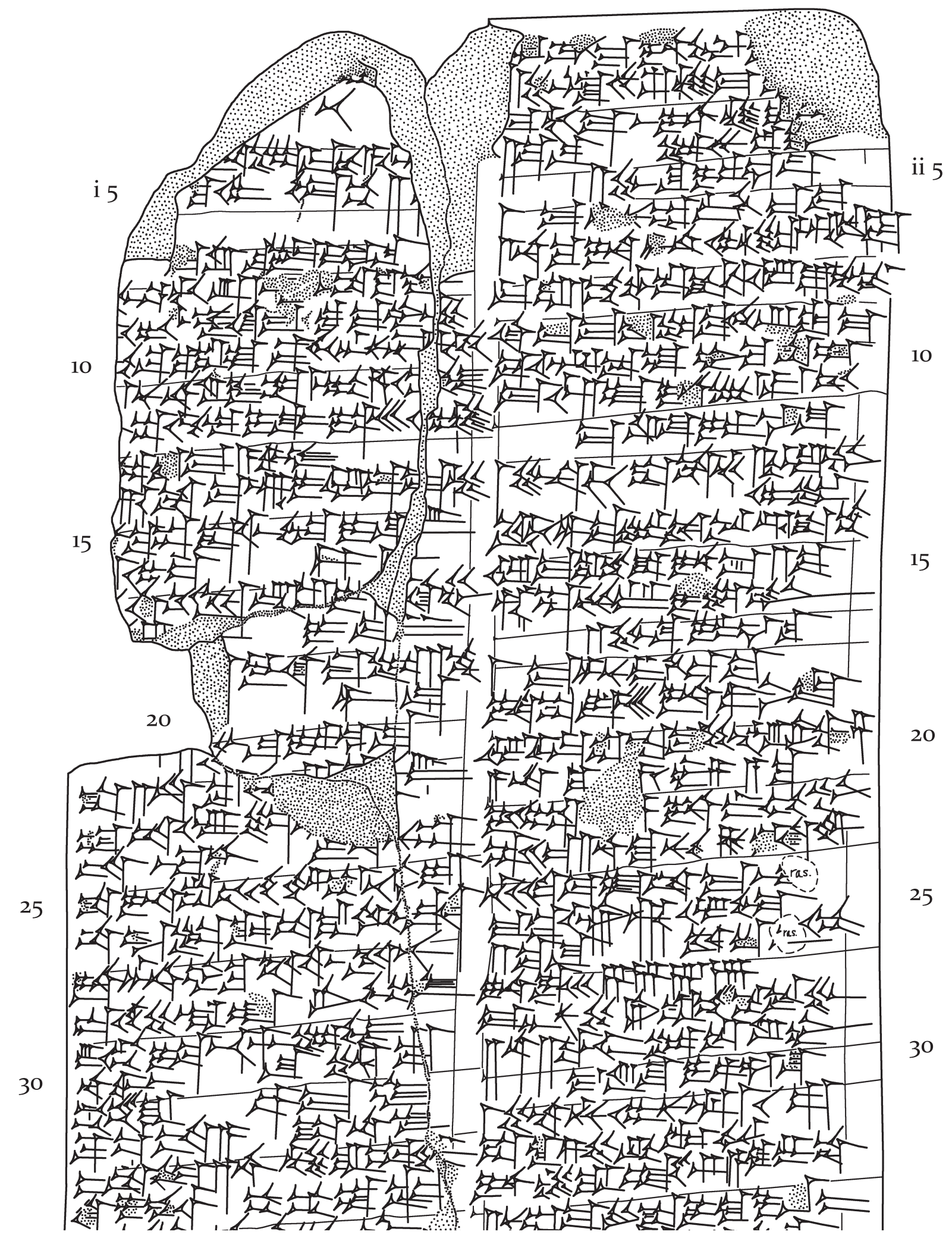

Fig. 1: YBC 2394 cols. i-ii, upper 


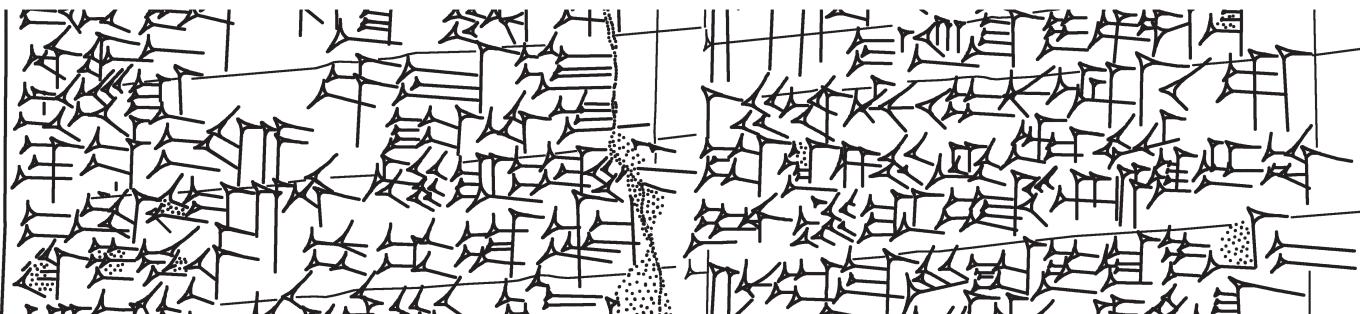

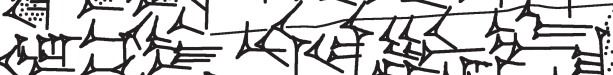

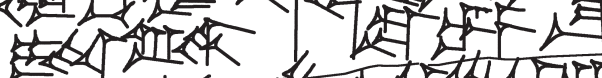

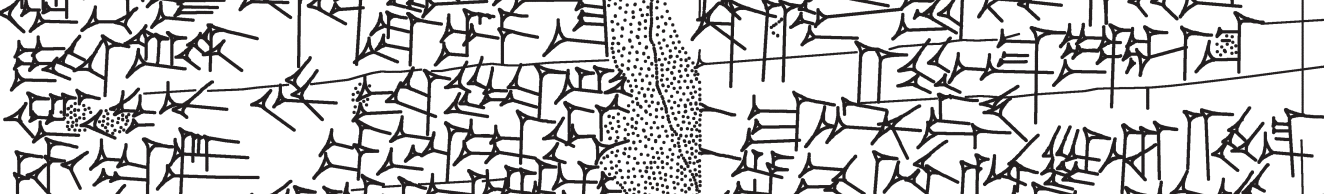
दिय

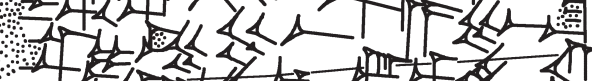
in

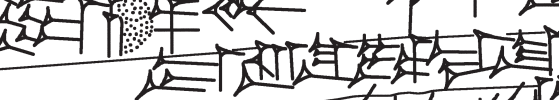

Fus , T 10 (1)

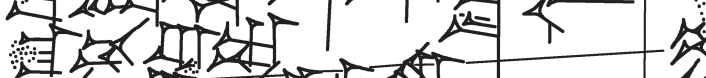
车近

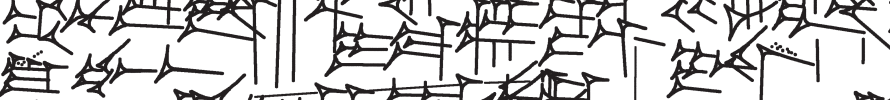

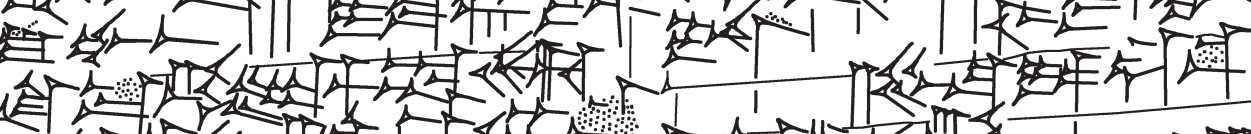

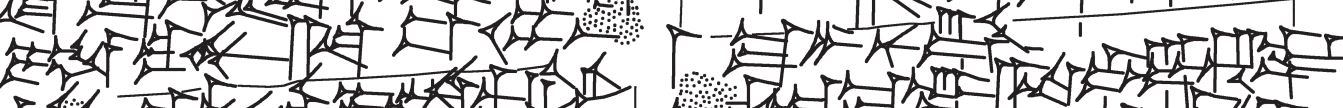

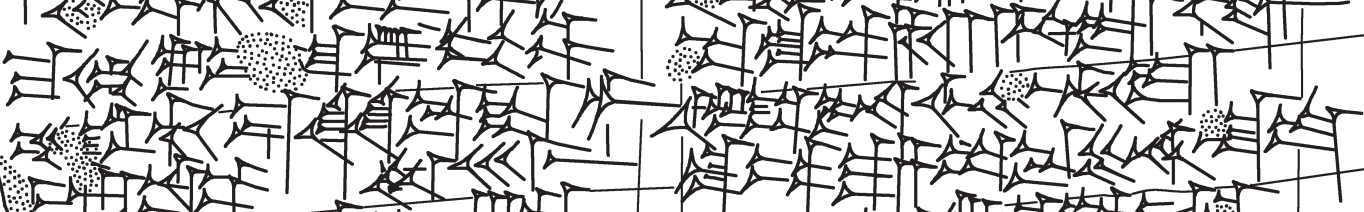
唯证 - 


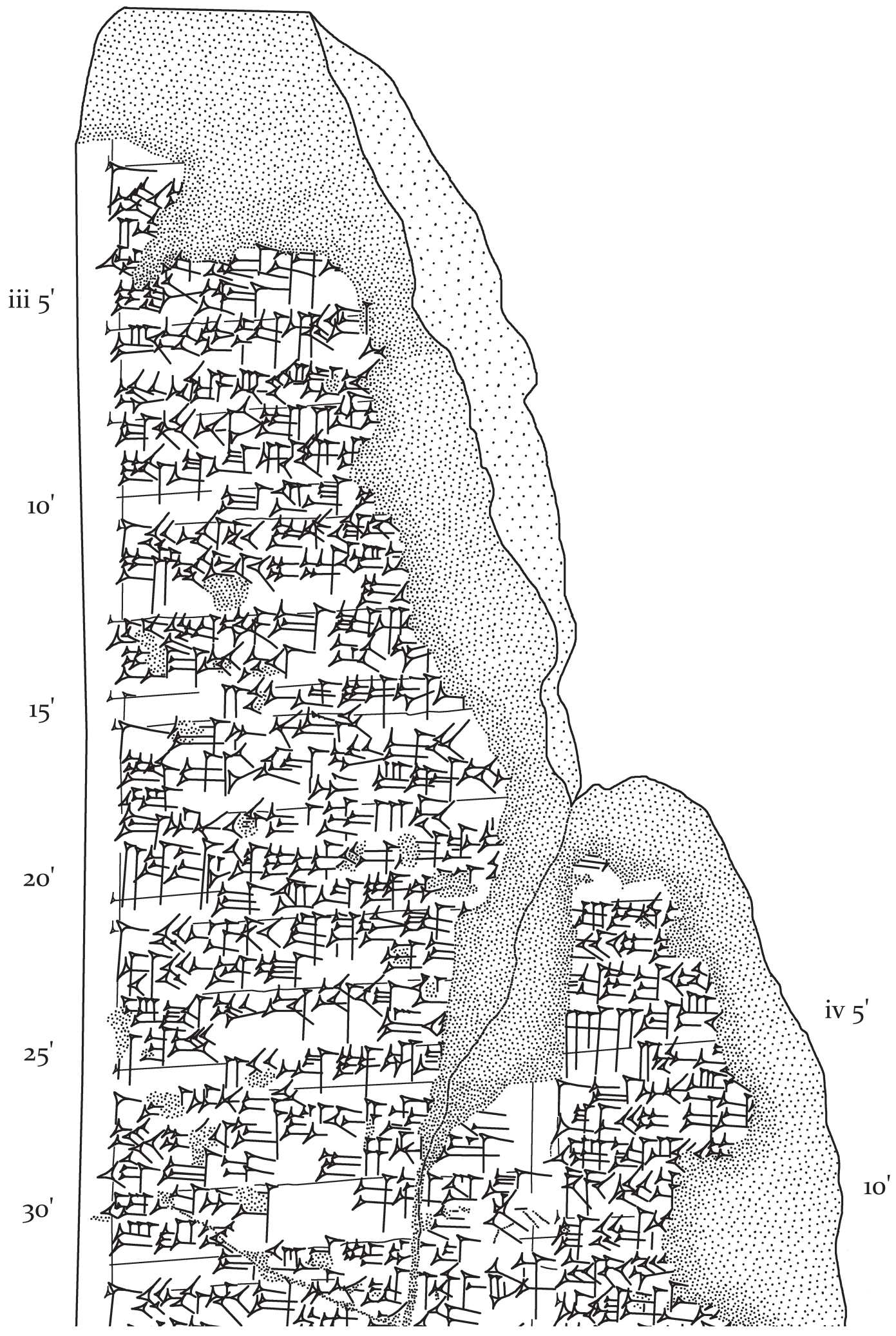

Fig. 3: YBC 2394 cols. iii-iv, upper 


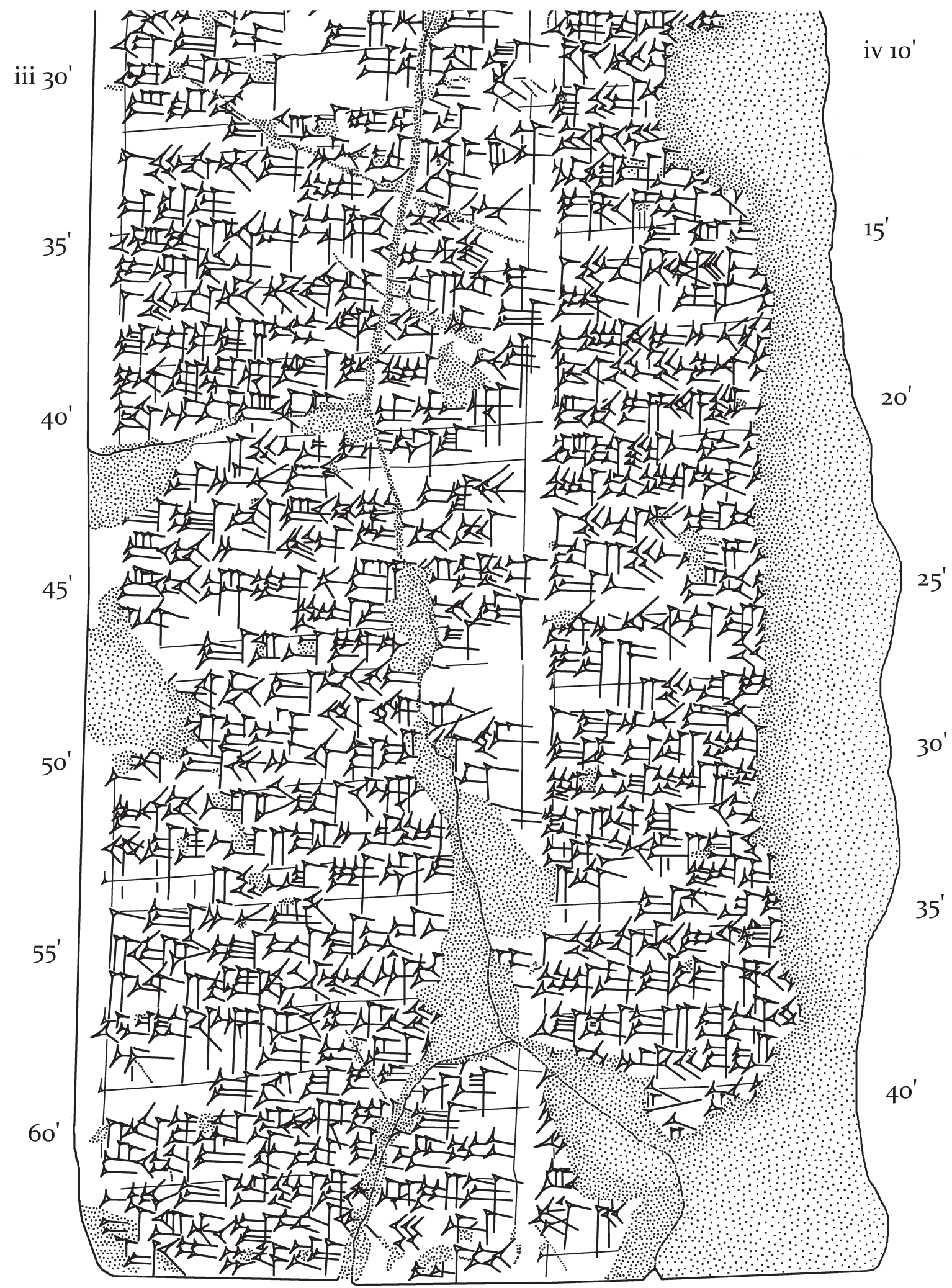

Fig. 4: YBC 2394 cols. iii-iv, lower 


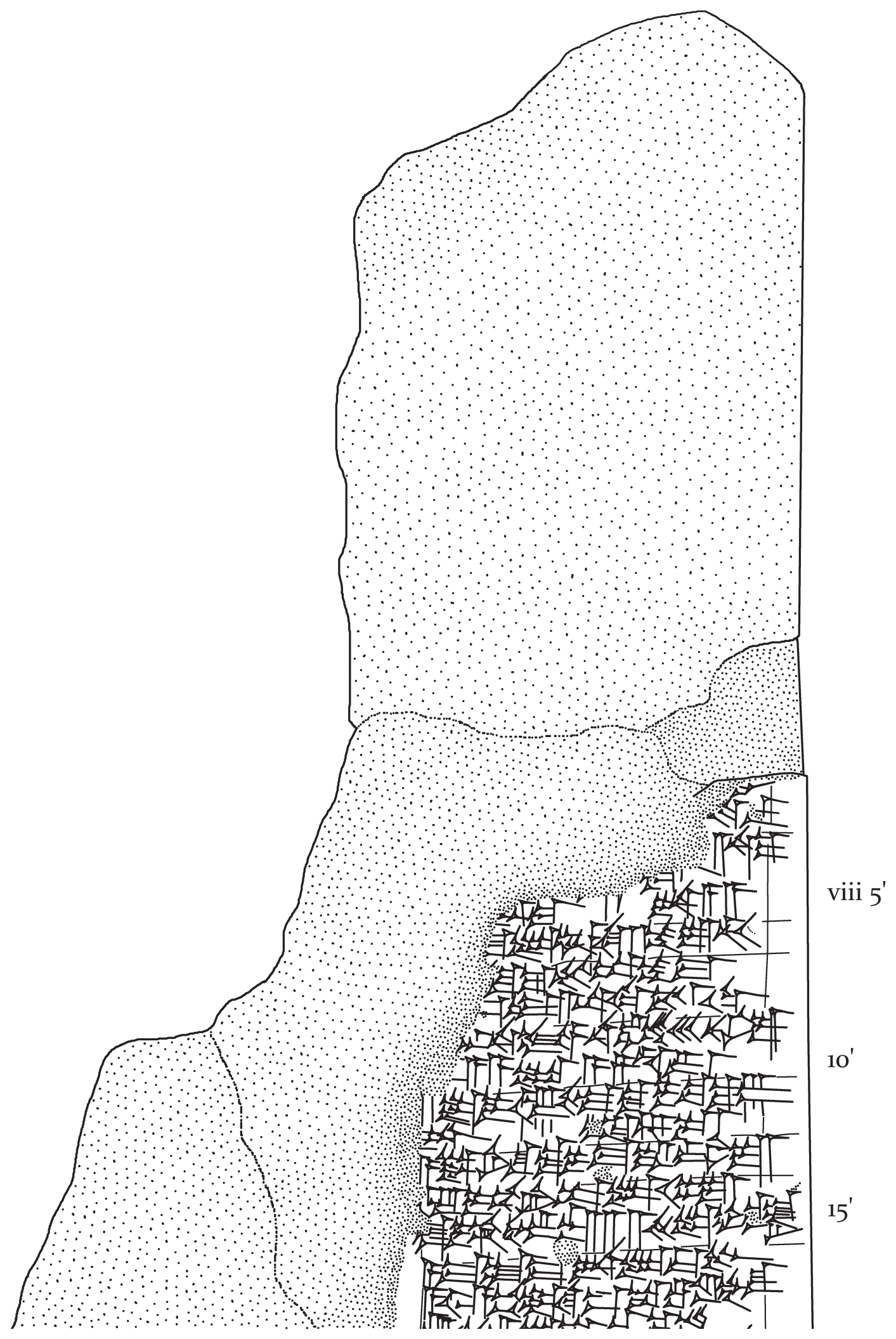

Fig. 5: YBC 2394 cols. vii-viii, upper 


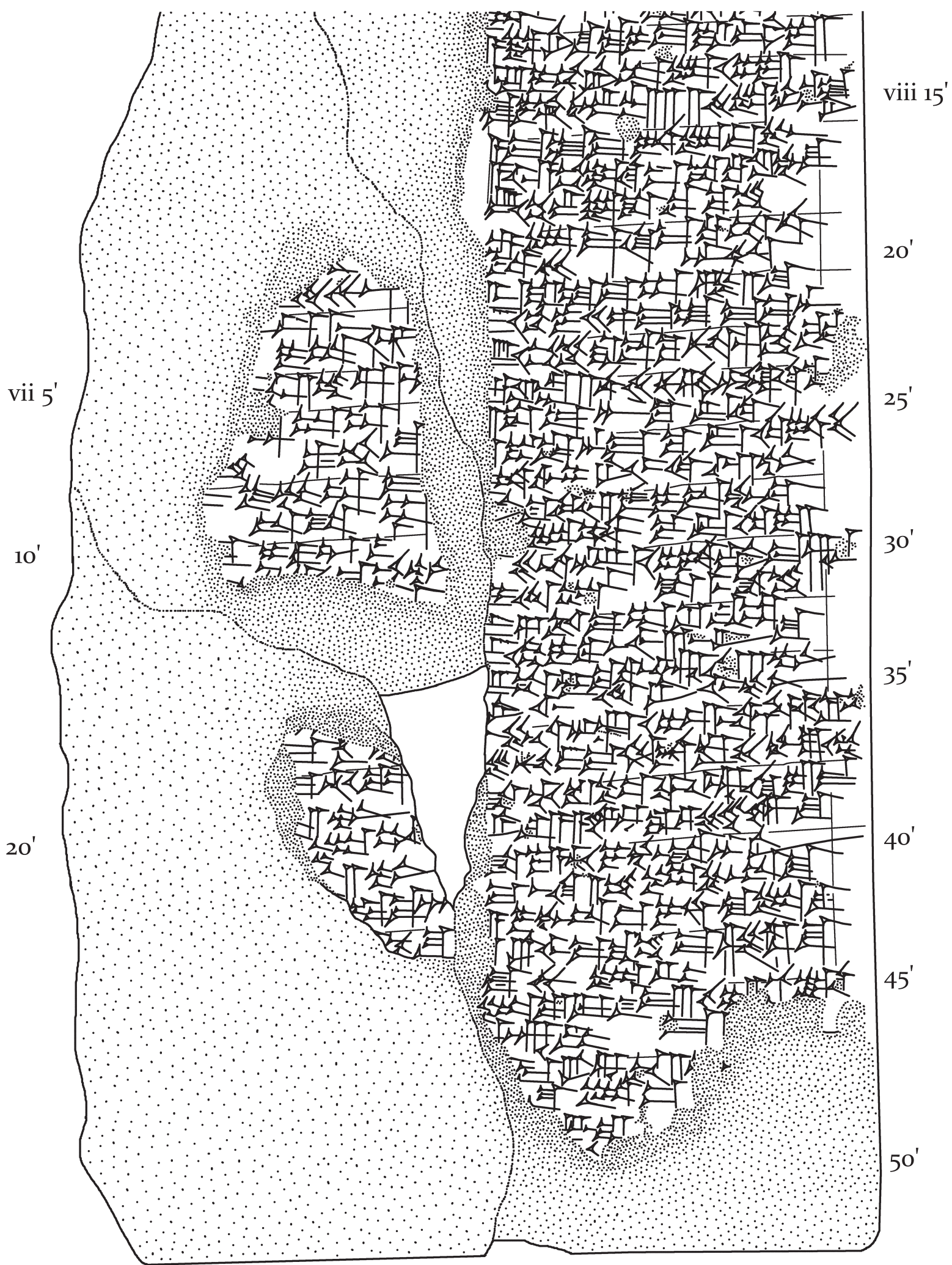

Fig. 6: YBC 2394 cols. vii-viii, lower 


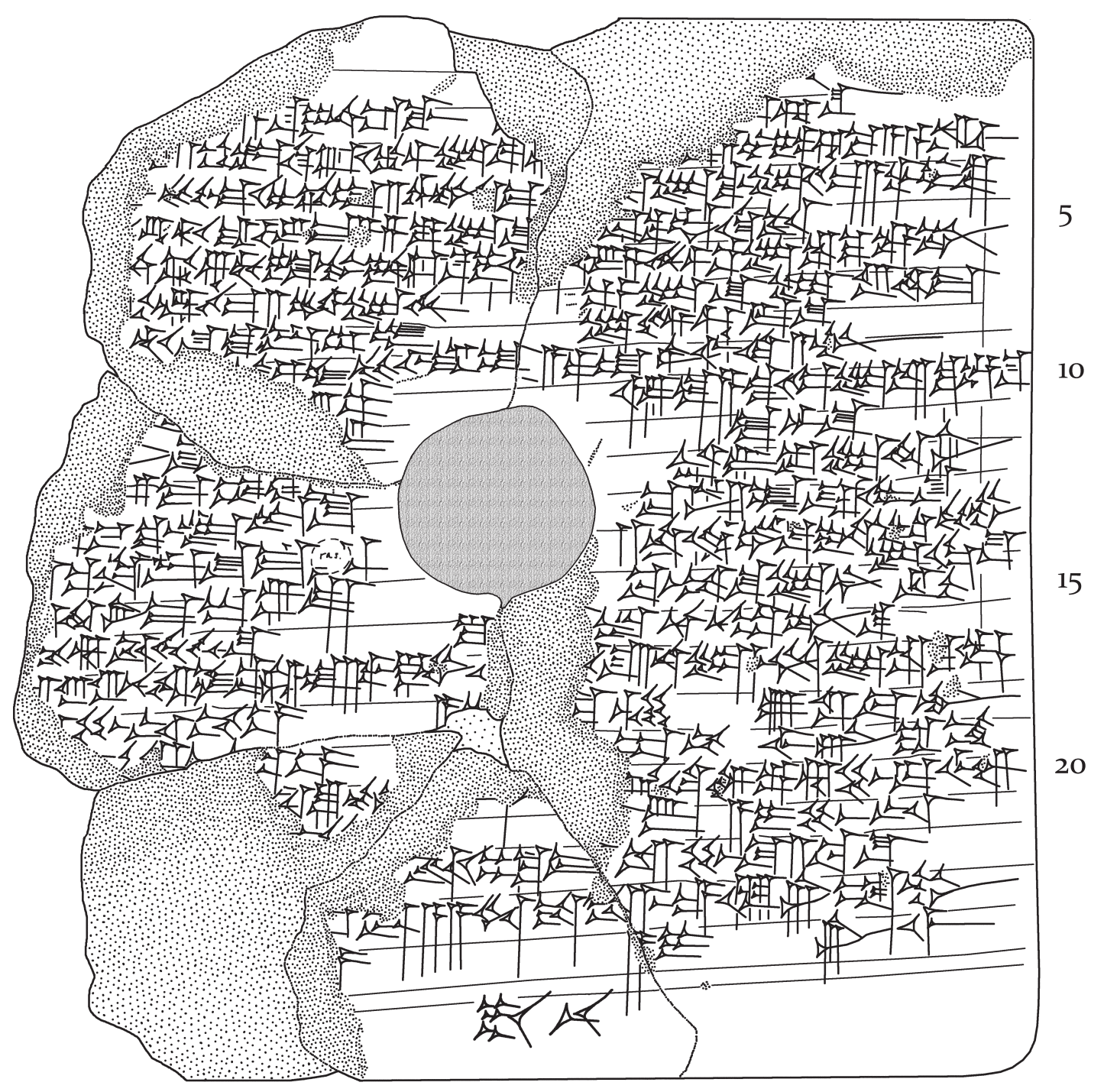

Fig. 7: YBC 2394 base 


\section{Photographs}
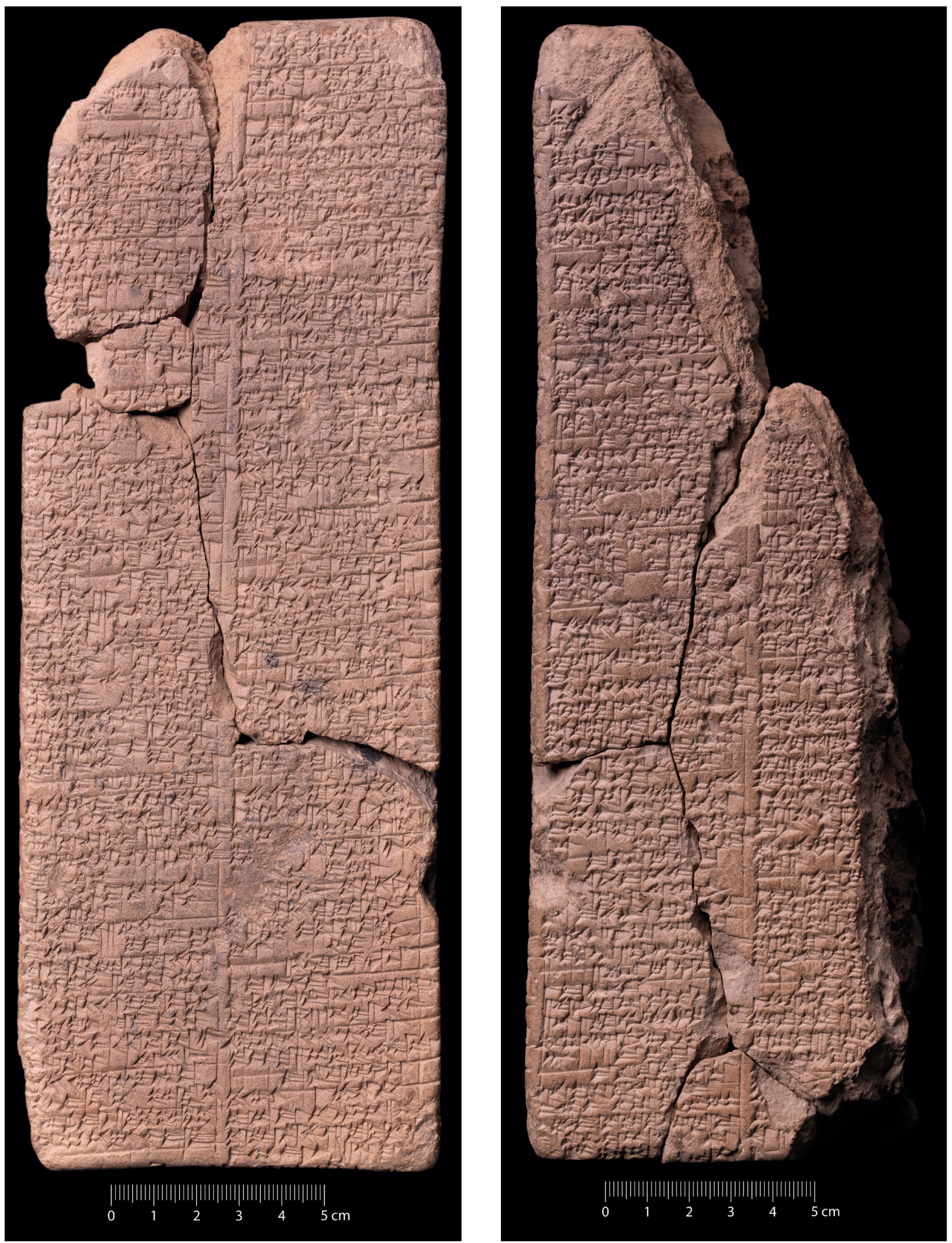

Fig. 8: YBC 2394 Side A

Fig. 9: YBC 2394 Side B 

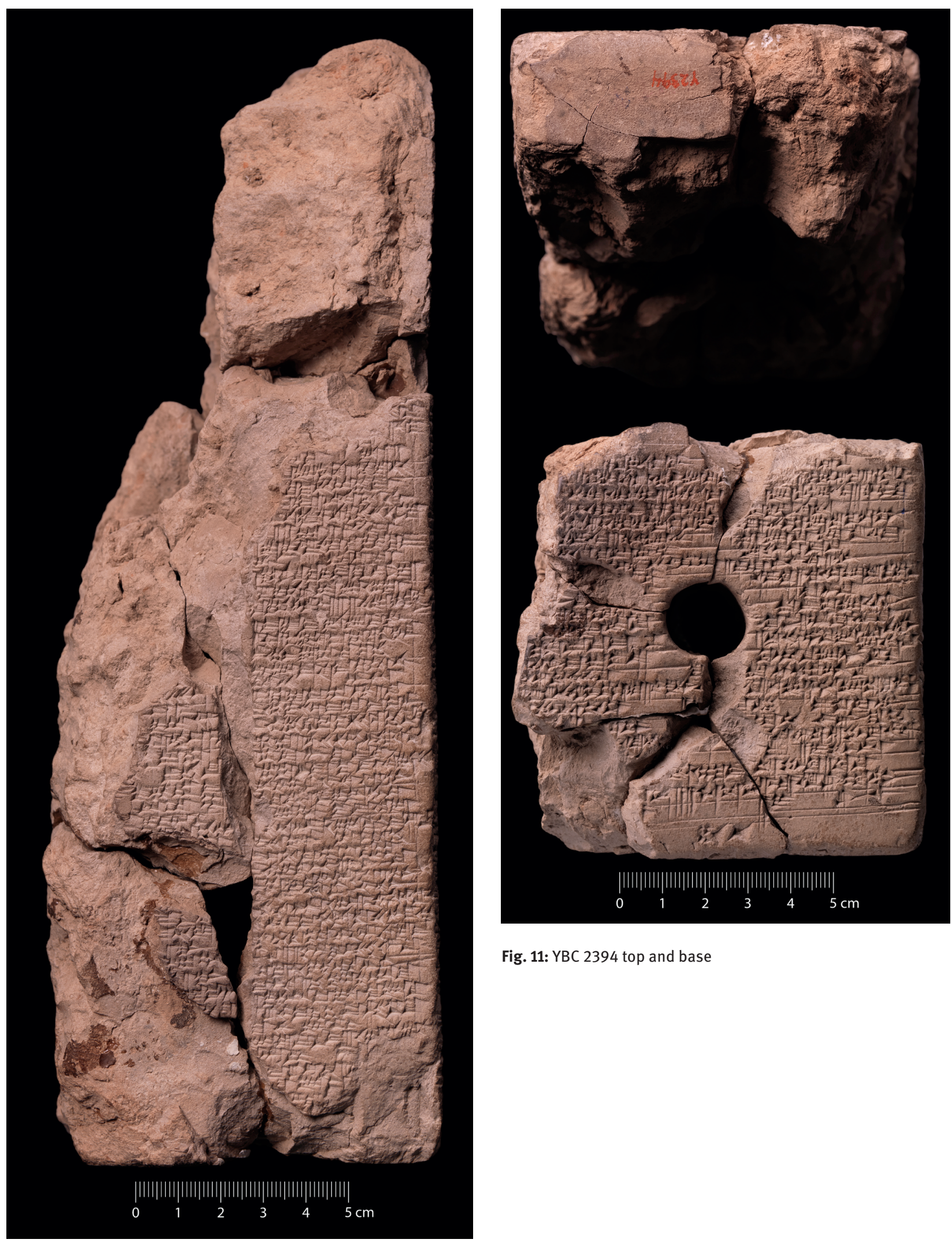

Fig. 11: YBC 2394 top and base

Fig. 10: YBC 2394 Side D 